\title{
versants
}

\section{Roorda au futur : écrits pédagogiques et école à venir}

\author{
Alain Ausoni \\ Université de Lausanne \\ ORCID: 000o-0o03-4970-5135
}

\begin{abstract}
Henri Roorda a beaucoup écrit sur l'école, et les critiques qu'il a formulées semblent avoir parlé à d'autres auteurs qui ont à leur tour dénoncé certains travers de l'institution scolaire de leur temps. Si une étude des spécificités poétiques et stylistiques des écrits pédagogiques de Roorda reste à faire, cet article se concentre sur les enjeux de ce qui semble être une de leurs particularités : le fréquent appui sur divers types d'anticipation. Singularisant son écriture combative, ces procédés contribuent au façonnement d'une posture d'acteur constructif et désamorcent le pathos de la controverse en la plaçant sur le temps long d'une histoire de progrès à venir. Voyons comment Roorda écrit au futur.
\end{abstract}

Keywords : Henri Roorda, pédagogie, futur, littérature romande, Edmond Gilliard, Denis de Rougemont.

«Dans cinquante ans, quand nous serons tous morts, les questions que j'agite dans mon petit livre se poseront encore. Que mes adversaires veuillent donc bien comprendre que ce n'est pas à leurs personnes que j'en veux.»

Henri Roorda, Avant la grande réforme de l'an 2000, 1925

(I970 : I06).

«Envers, en prose et contre tout. »

Henri Roorda, à ses élèves (Tapernoux 2017 : 138)

On peut être particulièrement touché par un objet d'études. Ayant découvert les écrits pédagogiques de Roorda à l'occasion d'un cours à l'Université de Lausanne, je leur ai consacré mon mémoire de licence. Après une formation pédagogique et quelques années d'enseignement au niveau secondaire, il m'est arrivé de m'y replonger pour réfléchir à ma pratique. Les lisant à nouveau dix ans plus tard, je constate sans m'en étonner qu'ils n'ont, pour moi, rien perdu de leur pouvoir. Dans le domaine de la littérature d'idées, on lit souvent les œuvres du passé d'abord en historien ou en ethnologue. On se frotte alors à l'altérité d'un monde, en s'offrant en retour l'occasion de faire le point sur la particularité du nôtre. Mais lisant Roorda désirant et dessinant une école différente, je me laisse séduire, et surtout convaincre. À plus de cent ans d'écart, l'efficace du discours est telle que 
je ne me dis pas : voilà ce qu'il aurait fallu faire pour changer l'école de ce temps-là. Je ressens un désir de changements pour l'école d'aujourd'hui.

\section{Pensée contagieuse, écrits singuliers}

Les écrits pédagogiques de Roorda n'ont pas laissé ses contemporains insensibles. Paru aux Cahiers vaudois en I9I7, Le Pédagogue n'aime pas les enfants a été beaucoup lu et, généralement, très bien accueilli (Enckell 2017 : 7-IO). Plus de quinze ans après son suicide, et alors même que Roorda avait assez rapidement cessé d'être actif dans les cercles anarchistes ou libertaires qui avaient publié ses premiers textes au tournant du siècle, l'article «Éducation » de l'imposant Dictionnaire de l'anarchisme publié en 1942 se termine par la citation d'une pleine page du Pédagogue, preuve de la postérité de sa pensée pour ceux qui mettaient au centre de leur combat la transformation des manières dont la société se charge d'instruire les enfants. La critique de certains défauts prêtés au système de l'instruction publique était un exercice commun dans le premier tiers du $\mathrm{xx}^{\mathrm{e}}$ siècle, pour une variété d'acteurs de tous bords politiques et répartis sur un large spectre socio-économique (Mole 2010 : 225-250). Pourtant, il n'est certainement pas exagéré de penser que la verve de Roorda a joué sa part dans la publication d'autres écrits contre l'école. Le jeune Denis de Rougemont s'appuie en tout cas explicitement sur Le Pédagogue n'aime pas les enfants, qualifié d'« excellent » et dont il « considère les thèses comme acquises » (Rougemont I984 : II9), pour écrire ses Méfaits de l'instruction publique (1929). Et les articles rassemblés en I942 par Edmond Gilliard pour composer L'École contre la vie doivent certainement aussi beaucoup à sa fréquentation de Roorda, son collègue au Collège cantonal et au Gymnase classique à Lausanne. Co-fondateur des Cahiers vaudois avec Paul Budry, Gilliard y avait prévu dès l'origine une place pour Le Pédagogue n'aime pas les enfants (voir Jakubec 2017 : I22), dont plusieurs idées - comme par exemple la prise de position sur la question de l'enseignement du latin - circulent dans son propre pamphlet. Certaines attaques contre l'école avaient d'ailleurs déjà été esquissées dans À Henri Roorda (I929), un livre-hommage présenté par Gilliard comme une poursuite de leurs échanges : « si j'écris ces lignes, ce n'est pas pour parler de toi aux autres, c'est pour parler avec toi. De nous » (Gilliard I973: I6).

Leurs liens intertextuels, la proximité de leurs dates de publication et l'intérêt continu pour les questions d'éducation en Suisse romande expliquent certainement que les Éditions L'Âge d'homme aient réuni en 1984 Le Pédagogue n'aime pas les enfants, L'École contre la vie et Les Méfaits de l'instruction publique dans un volume intitulé Trois pamphlets pédagogiques. Reprenant une formule peu modeste de Denis de Rougemont à propos de son propre texte, l'éditeur dit de ces trois écrits qu'« ils n'ont pas vieilli » (qua- 
trième de couverture). S'étonnera-t-on si je soutiens ici que le texte de Roorda semble résister mieux que les deux autres à l'épreuve du temps ? Peuton juger fécondes aujourd'hui les attaques du jeune Rougemont contre le " principe de l'instruction publique » (Rougemont I984 : I2I) ou contre « cet attentat à l'intégrité humaine qu'est en fait l'esprit démocratique » (I20) ? Son texte ne nous en apprend-il pas plus sur la situation d'un jeune homme de vingt-deux ans, désireux de se faire une place dans le monde des lettres en expérimentant "une liberté d'écriture et des audaces argumentatives qui feront l'admiration de ses lecteurs » (Mole 2019: 63), que sur l'école ? Participant du façonnement de diverses postures littéraires, le discours sur (mais souvent contre) l'école est un topos du récit de l'entrée en littérature et de la définition du rapport d'un écrivain à sa langue, comme en témoigne par exemple, pour le contexte culturel qui nous intéresse, le Raison d'être de Ramuz (Jakubec 2017 : I22). De ce point de vue, alors âgé de soixante-six ans (dont trente passés à enseigner), Gilliard saisit l'occasion de son École contre la vie pour se présenter en écrivain toujours mû par l'énergie et les idéaux de son enfance :

J'ai cette chance encore, à plus de soixante ans, de sentir les plus mûres, les plus méditées indignations de ma vieillesse passionnément solidaires des instinctives révoltes de mon enfance.

Je ne parle pas de mon enfance par souvenir, ce qui la relogerait dans l'autrefois. Il ne s'agit pas d'un rappel du passé ; il s'agit d'une revendication du présent. [...] Mon enfance ne s'est jamais résignée. Elle attendait le moment où la vie, tout avertie, mettrait à son service les moyens de la revanche sûre. Elle attendait son heure d'autorité. Chez ceux qui ne renient rien d'euxmêmes, les impatiences de l'enfance et de l'adolescence trouvent leur soulagement (leur "application") dans les urgences souveraines de la vieillesse qui a conquis, par le risque de toute la vie, la sereine propriété de l'audace, le sommaire pouvoir exécutif de la liberté (Gilliard I984 : 82).

Gilliard pare du même coup tout reproche sur sa complicité au système qu'il pourfend : il en a souffert aussi en tant qu'enseignant et a dû attendre la maturité nécessaire aux « moyens de la revanche sûre » pour pouvoir l'exprimer avec force et justesse. Mais Roorda est à n'en pas douter celui des trois auteurs qui parle le moins de lui dans ses écrits sur l'école, une disposition qui converge sans doute avec son choix de l'écriture pseudonymique pour son activité de chroniqueur et avec sa propension à ponctuer ses écrits sur l'école de scènes fictionnelles à valeur d'exemples autant que de tranches de vie. Moins tournées vers l'expression d'une personnalité ou d'un art poétique, ses thèses sur l'école jouissent ainsi certainement d'un plus haut degré d'universalité. 


\section{Roorda au futur}

Une étude stylistique des écrits pédagogiques de Roorda, propre à faire le point sur les mécanismes de la persuasion, reste largement à faire. Au risque d'essentialiser sa pensée et les modes de sa communication littéraire, elle se devrait d'être attentive aux évolutions qui caractérisent une pratique se développant sur les près de trente ans qui séparent « L'École et l'apprentissage de la docilité » (I898) d'Avant la grande réforme de l'an 2000 (1925). Car au cours de cette période historique troublée, où l'instruction obligatoire alimente un large débat public et fait l'objet de nombreuses expérimentations, plusieurs événements et expériences n'ont pu manquer d'influencer la pensée pédagogique de Roorda sur l'école et son ethos de critique, donnant à ses textes des formes et des tonalités toujours renouvelées. Considérant l'évolution de l'œuvre pédagogique de Roorda, Gilles Losseroy juge qu'elle « s'est fait moins radicale (mais non moins pénétrante) avec le temps » (2009: 58). Cela tient certainement au changement voire à l'élargissement du lectorat visé : d'articles pour des revues anarchistes ou libertaires françaises, Roorda passe à des livres édités en Suisse romande, à destination d'un public intéressé par la question de l'école, mais aux sensibilités politiques plus hétérogènes. Quoi qu'il en soit, pour étayer l'impression de Losseroy, on peut relever certains énoncés réflexifs et certaines modulations dans l'usage d'expressions traversant ses écrits qui marquent des évolutions dans la pensée de Roorda ou des changements dans les stratégies mises en œuvre pour la communiquer. Dans les premières pages d'Avant la grande réforme de l'an 20oo, publié l'année de sa mort, Roorda revient ainsi par exemple sur ses premiers écrits pédagogiques :

Il y a vingt-cinq ans, j'aurais prouvé que mon système était conforme aux lois de la Nature et à la Raison. Mais le goût de ces facéties m'a complètement passé. Que veut la Nature? Je n'en sais rien; et elle ne le sait sans doute pas mieux que moi (Roorda 1970 : I09).

Assumant sa subjectivité, et relevant les biais induits par sa position dans le système qu'il dénonce, Roorda place ouvertement le débat sur un plan idéologique. L'analyse de la question scolaire dépend de l'idée qu'on se fait de la société, et de son évolution, ainsi que de notre représentation des besoins des élèves. Il ne s'agit plus seulement de dénoncer, sur un mode parfois carnavalesque, une institution dont l'action serait contre nature ou déraisonnable dans l'absolu, mais d'imaginer des manières d'infléchir les pratiques pédagogiques. On peut dès lors se demander si, dans la typologie de la littérature communément dite "de combat", les derniers textes pédagogiques de Roorda relèvent bien du pamphlet (voir Angenot 1982 : 27-45). Contrairement à Rougemont (I984 : I62), Roorda n'utilise en tout cas pas le 
mot dans les textes concernés. Qualifiant son dernier écrit sur l'école de « livre de propagande » (Roorda 1970 : I06), il cherche à provoquer le succès de ses idées et à modifier des conduites, plutôt qu'à faire éclater un scandale. Il semble en effet bien difficile de faire aux derniers écrits pédagogiques de Roorda les reproches adressés au pamphlet de Gilliard lors de sa publication, qui vaudraient largement aussi pour celui de Rougemont :

\begin{abstract}
Mieux vaut besogner même humblement dans la réalité, que d'évoquer éloquemment une révolution idéale dont on ne dit même point ce qu'elle sera. Pour être avec la vie, il faut composer avec elle. L'école actuelle est peut-être contre la vie. Elle le sera bien davantage encore si, à l'instar de M. Gilliard, on néglige de se vouer à des réformes possibles pour se plaire à un savant mais combien stérile démolissage (Braichet $1943:$ I).
\end{abstract}

Comme membre du comité de la Ligue internationale pour l'éducation rationnelle de l'enfance fondée en Espagne par le pédagogue Francisco Ferrer, comme auteur de manuels de mathématiques pour les élèves suisse romands, par son implication dans l'expérience de l'école Ferrer de Lausanne (I9IO-I9I9) et son activité dans la Société suisse des professeurs de mathématiques (voir Enckell 20I7 : IO-I5), Roorda se fait fort de « besogner dans la réalité ». Ses observations de la vie scolaire n'ont d'ailleurs qu'exceptionnellement été remises en cause. Bien plus, Roorda dessine certains aspects concrets d'une école à venir, une manifestation parmi d'autres de son penchant pour «la recherche du futur », dont Gilliard a été le témoin admiratif:

Il y en a qui "sentent" d'ici les terres de l'au-delà ; qui perçoivent avant les autres l'odeur des nouveaux rivages du temps. Ils partent à la recherche du futur comme d'autres sont partis à la recherche d'Indes nouvelles (Gilliard $1973:$ I3).

Dans ce qui suit, je m'intéresserai à la manière dont divers types d'anticipation sont mis en scène dans Avant la grande réforme de l'an 2000. Caractéristiques des derniers écrits pédagogiques de Roorda, et contribuant sans doute à leur efficacité particulière, ces procédés participent du façonnement d'une posture d'acteur constructif et désamorcent le pathos de la controverse en la plaçant sur le temps long d'une histoire d'inéluctables progrès à venir. Ils préparent ainsi un terrain propice au déploiement de sa verve humoristique. Si cette approche ne me permettra pas de considérer en détail la pensée pédagogique de Roorda (voir à ce sujet Lenoir 2009 et L'Aminot 2009), j'espère néanmoins faire découvrir, au passage, certaines de ses réflexions, sans doute moins connues que celles du Pédagogue n'aime pas les enfants, qu'Avant la grande réforme de l'an 2000 prolonge en quelque sorte. Composé à une période où Roorda savait peut-être déjà qu'il en fi- 
nirait bientôt, ce texte peut se lire, on l'a vu, comme une forme de bilan de ses écrits sur l'école, rendant manifeste certaines évolutions de sa pratique critique. Voyons donc comment Roorda écrit au futur.

\section{Prolepse argumentative}

Dès son titre, Avant la grande réforme de l'an 2000 oblige le lecteur à se projeter dans un avenir qui aura fait un sort aux problèmes pédagogiques dénoncés. Ses lecteurs savent que cela n'empêche bien sûr pas Roorda de s'impatienter face à la « lenteur désespérante » de tout progrès dans le monde scolaire (Roorda I970 : I06) ou d'afficher parfois un certain pessimisme quant à l'effet de son combat pour une école meilleure (voir Lenoir 2009: 53-56), l'une des deux plus longues sections de ce texte s'intitulant significativement «Scepticisme » (Roorda I970 : I06-II6). Par la longueur de l'attente anticipée, il ne se prive ainsi pas de pointer la difficulté de toute réforme de l'institution scolaire, qu'on impute communément à sa taille, à sa complexité et aux réticences des fonctionnaires qui l'animent (Prost 2013 : 30I). Mais en dessinant néanmoins l'horizon de cette réforme, Roorda s'extirpe de la "vision crépusculaire du monde » qui gouverne l'écriture pamphlétaire : à la fois impératif (face à la prédominance de l'imposture, les effets néfastes de l'erreur) et inutile (étant donné la « perversion accomplie des valeurs »), le discours du pamphlet s'entend typiquement comme " une voix d'après le désastre » (Angenot $1984: 344$, 346). C'est ainsi que Rougemont appelait assez brutalement des changements radicaux pourtant jugés impossibles et que Gilliard s'enfermait de la même manière dans une forme de pessimisme contemplatif : « l'école ne bougera pas. On peut, à certains moments, croire qu'elle se met en marche. Elle tourne seulement sur son pivot et ramène sa face inchangée. Elle dispose d'une souveraine puissance : l'inertie » (Gilliard I984 : I09). Roorda postule, lui, qu'une réforme finira par se faire. C'est donc qu'on aura saisi les effets pervers de la situation antérieure, lesquels doivent être nombreux puisque la réforme de l'an 2000 sera « grande ». La durée considérée doit servir à ménager les egos et permettre, dans l'idéal, un diagnostic dépassionné :

Dans cinquante ans, quand nous serons tous morts, les questions que j'agite dans mon petit livre se poseront encore. Que mes adversaires veuillent donc bien comprendre que ce n'est pas à leurs personnes que j'en veux (Roorda I970 : I06).

Commune dans le débat d'idées, la prolepse provoque un exercice de pensée consistant, pour toute personne concernée par l'institution scolaire, et en particulier ses responsables et les enseignants, à soutenir le regard 
des réformateurs du futur. Se pose du même coup la question de l'attitude à adopter pendant les septante-cinq années qui s'écouleront d'ici la réforme annoncée. Pour ne pas être jugés trop négativement par les réformateurs du futur, pourrait-on mettre en œuvre certains changements favorables à l'épanouissement des élèves ? Il s'agit ainsi pour Roorda de préparer les mentalités et de proposer des inflexions que les praticiens pourront mettre en œuvre dans l'intervalle.

\title{
Avenir des élèves et école à venir
}

En I886, dans une brochure intitulée L’Avenir de nos enfants, le célèbre penseur anarchiste Élisée Reclus signalait à ses camarades, engagés prioritairement dans d'autres luttes, l'importance particulière d'une réforme du système éducatif pour l'avènement d'une société plus égalitaire :

\begin{abstract}
Maintenant que l'école est laïque, la formule religieuse a été remplacée par une formule de grammaire, les sentences latines incompréhensibles ont fait place à des mots français qui ne sont pas plus clairs. Que l'enfant comprenne ou non, peu importe ; il faut qu'il apprenne selon un formulaire tracé d'avance. [...] D'ailleurs, ces écoles sont-elles sans esclavage, sans heures de retenue et sans barreaux aux fenêtres? Si l'on veut une génération libre, que l'on démolisse d'abord les prisons appelées collèges et lycées (Reclus I886 : 5).
\end{abstract}

Les liens de Reclus et d'autres penseurs anarchistes ou libertaires avec le jeune Roorda (et sa famille), comme leur fraternité d'esprit, sont connus (voir Losseroy 2009) et expliquent que la pensée anarchiste de l'éducation irrigue très manifestement ses premiers textes pédagogiques : quel que soit le détail des aspects considérés, Roorda attend de l'instruction publique qu'elle joue son rôle dans l'avènement d'une société plus égalitaire, dont bénéficieront les écoliers devenus citoyens. La question de l'avenir des élèves est posée un peu différemment dans Le Pédagogue n'aime pas les enfants et Avant la grande réforme de l'an 200o. Beaucoup des propositions de ces textes découlent d'un principe simple, trop souvent ignoré des pédagogues selon Roorda : "nous ne savons pas ce que sera la vie de l'écolier qui est là, devant nous " (Roorda 1969: 282) ou, en d'autres termes, huit ans plus tard, " le maître s'adresse à des enfants qui exerceront, par la suite, les professions les plus diverses ; à des enfants dont l'avenir est, pour lui, absolument indéterminé » (Roorda 1970 : II9). De là son combat pour une longue formation commune et généraliste, qui irait à l'encontre de ce qu'on nomme aujourd'hui en Suisse romande (comme par euphémisme) "l'orientation" et qui, de son temps, déterminait précocement et assez irrémédiablement la possibilité de l'accès aux études dites "secondaires". La pertinence du prin- 
cipe de cette « école unique » (II9-I22) ne serait d'ailleurs pas remise en cause si la future profession des élèves pouvait être prédite :

Considérons le cas exceptionnel où l'on peut prévoir, avec une sûreté presque absolue, le métier que l'enfant exercera plus tard. Jean succédera à son père, le chapelier. Il est actuellement sur les bancs de l'école primaire. Pierre, qui vient de commencer ses études classiques, sera un jour avocat. Y a-t-il une raison quelconque pour que le premier, avant le jour où commencera sa préparation professionnelle, reçoive une éducation intellectuelle différente de celle qu'on va donner au second ? S'il existe une pédagogie particulièrement propre à assouplir et à fortifier les jeunes intelligences, faut-il ne l'appliquer qu'à l'un de ces deux enfants?

Dans un chapelier il y a quelque chose de plus qu'un chapelier ; dans un avocat il y a quelque chose de plus qu'un avocat. Pierre et Jean, très probablement seront, un jour, pères de famille. En qualité de citoyens, ils prendront part aux mêmes luttes politiques et ils auront les mêmes questions à résoudre. Est-ce le chapelier ou est-ce l'avocat qui sera député ? Personne ne saurait le dire. Et il pourrait arriver que Jean fût plus intelligent et plus facilement "cultivable" que Pierre (I2I).

L'école devrait former au mieux tous les élèves, sans pouvoir faire comme si leur vie future se limitait à leur activité de travailleur. Roorda ne manque pas de pointer les mécanismes de la reproduction sociale, et l'école qu'il défend doit contribuer à en réduire les effets. Mais plutôt que d'envisager la question sous l'angle idéologique à l'échelle de la société, il se met au niveau des élèves, dans la singularité de leurs trajectoires existentielles : « l'école unique », telle qu'il la conçoit, « fournira à chaque enfant l'occasion d'améliorer ce que la nature lui a donné de bon » (I2I-I22). Vaste programme, concrétisé dans les derniers écrits de Roorda sur l'école, dont Lenoir synthétise ainsi les différentes dimensions : une pédagogie de l'intelligence et de l'activité ; une pédagogie ludique et de l'intérêt ; une pédagogie de la recherche ; une pédagogie individualisée et des représentations ; une pédagogie pragmatique (Lenoir 2009 : 27-46). Pour dessiner certains principes et surtout certaines pratiques pédagogiques qui gagneraient à être mis en œuvre, Roorda s'appuie dans ces textes sur de nombreux énoncés au futur simple, laissant ainsi entendre que leurs conditions de réalisation sont peu ou prou remplies. Nulle utopique réforme en vue, mais des perfectionnements qui ne demandent qu'à se réaliser pour le bien des écoles : « on pourra les améliorer beaucoup dès qu'on le voudra réellement » (Roorda I969: 225).

Contrairement à Rougemont et à Gilliard, Roorda n'attaque pas frontalement le principe de l'instruction publique ni ne cible d'abord des effets de système dans ses derniers écrits sur l'école. Le titre de son Pédagogue n'aime pas les enfants l'indiquait déjà : il cherche à secouer les acteurs du monde scolaire, et en particulier les enseignants. De même, la préface à Avant la grande réforme de 
l'an 2000 anticipe des troubles dans la réception, et identifie par là ceux qui se sentiront malmenés : il est prévu que le livre fera « de la peine à quelques pédagogues, qui depuis longtemps, accomplissent leur tâche très consciencieusement » (Roorda I970 : I05). Roorda semble ainsi juger que les enseignants, par leurs poids dans l'institution et le degré de liberté dont ils jouissent en pratique, pourront contribuer "de l'intérieur" à changer l'école. S'adressant aussi à eux, il oscille entre la formulation de propositions réalisables sans trop toucher au cadre scolaire de son temps et, occasion privilégiée d'exercer sa verve humoristique, la dénonciation d'habitudes jugées néfastes.

On a peut-être peiné à considérer ensemble la hargne du pédagogue libertaire et les facéties du chroniqueur - pour évoquer le sous-titre de la première monographie consacrée à Roorda (Roorda 2009) qui identifie à raison, mais sépare du même coup, deux pans de son activité littéraire. Or il faudrait montrer qu'elles fonctionnent de concert dans certains passages de ses écrits sur l'école. Ce serait l'objet d'un autre article, mais voyons pour finir comment Roorda s'appuie sur une prédiction pour dénoncer le problème de l'encyclopédisme des programmes scolaires et comment il cherche par l'humour à les défétichiser. Prédisant avec raison les développements de l'accès au livre et l'essor ce qu'on appellera la "formation continue", Roorda explique que les années d'école ne devraient plus être considérées comme l'unique occasion d'acquérir certaines connaissances : "la société moderne offrira toutes sortes de facilités à celui qui, après ses années d'écoles, voudra continuer à accroître ses connaissances » (Roorda I970 : II7). C'est une raison de plus de souhaiter que « les faits enseignés fussent moins nombreux et mieux choisis, que le maître ne fût pas pressé et que l'écolier, moins vite défraîchi, eût le temps de penser » (II7). Les lecteurs de Roorda connaissent ses inlassables charges contre « le savoir inutile » et le " bourrage des crânes ", expressions récurrentes dans ses écrits scolaires. Dans son dernier texte sur l'école, mettant les enseignants face à leurs propres lacunes dans des matières scolaires dont ils ne sont pas spécialistes, il leur propose de conserver leur sérénité « lorsqu'un collégien de quatorze ans se permet, par exemple, de confondre Philippe-Auguste avec Louis-Philippe » et de s'investir hardiment pour mettre fin au problème :

Si les professeurs de littérature étaient chargés de faire des coupes sombres dans les cours de mathématiques ; si les géomètres étaient priés de bien vouloir simplifier les cours d'histoire; et si les historiens avaient le droit de ramener à la modération leurs collègues des sciences naturelles, un grand progrès serait bientôt réalisé. Et, pourtant, tous ces hommes reconnaissent la grande valeur de l'instruction (II7).

Comment ne pas sourire, ni se demander si cela ne vaudrait pas aussi un peu pour l'école d'aujourd'hui? 


\section{Et maintenant?}

En Suisse romande, aucune réforme scolaire d'envergure ne semble avoir particulièrement marqué l'an 200o. L'école d'aujourd'hui diffère pourtant significativement de celle que connaissait Roorda. D'importants changements ont en particulier été permis et nourris par la démocratisation des études supérieures, si bien que la deuxième partie de cette citation de son premier écrit publié sonne aujourd'hui comme une évidence pour beaucoup :

Si telle méthode d'éducation doit accroître cette vie, la rendre plus profonde et plus heureuse, nous pensons qu'elle est bonne pour le futur paysan comme pour le futur historien, et bonne aussi pour celles qui seront plus tard leurs compagnes (Roorda I898).

Mais qu'en est-il de la première partie? Avons-nous construit l'école dans laquelle l'enfant pourra s'épanouir ? L'intérêt contemporain pour les textes de Roorda, dont ce numéro comme diverses rééditions récentes témoignent, ne signale-t-il pas une impossibilité de répondre vraiment par la positive? Contrairement à ce que prédisait Roorda dans Avant la grande réforme de l'an 200o, ses écrits sur l'école n'ont pas « sombré, comme beaucoup d'autres ouvrages, dans l'océan des imprimés " (Roorda 1970 : II6). On a même pu souhaiter que Le Pédagogue n'aime pas les enfants figure obligatoirement parmi les lectures de tous les enseignants en formation (L'Aminot $1997: 217$ ). Deux prémisses, sans doute, à cette proposition. La première est un constat : beaucoup des changements de l'école ne sont pas le fruit de réformes datables et imposées par les pouvoirs publics, mais résultent d'évolutions sociales affectant au fil du temps les pratiques de terrain, si bien qu'ils finissent par s'imposer avec la force de l'évidence (Prost 2013 : 30I-304). Roorda semblait en être conscient, lui qui ciblait particulièrement les enseignants. Proposant des aménagements plutôt que des réformes, il travaillait les mentalités par sa verve humoristique. La deuxième prémisse voudrait que, de nombreuses propositions de Roorda n'ayant pas perdu de leur pertinence ni ses textes de leur pouvoir persuasif, leur lecture puisse être un opérateur de ce type de changements. Il n'y aurait alors pas grand risque à parier que sa pensée de l'école continuera d'agir : on Roordera. 


\section{Bibliographie}

Angenot, Marc, La Parole pamphlétaire. Typologie des discours modernes, Paris, Payot, I982.

Braichet, René, «À propos d'un récent pamphlet. L'École contre la vie », Feuille d'avis de Neuchâtel, 30 avril I943, p. I.

Enckell, Marianne et al., Les Anarchistes : dictionnaire biographique du mouvement libertaire francophone, Ivry-sur-Seine, L'Atelier/Ouvrières, 2014.

— «Préface ", Le Pédagogue n'aime pas les enfants, Lausanne, HumuS/Association des amis de Henri Roorda, 20I7, pp. 7-I7.

Fonjallaz, René, À la mémoire de Henri Roorda, Genève, René et ses amis, 1928.

Gilliard, Edmond. À Henri Roorda [1929] et L'École contre la vie [1942], Lausanne, Bibliothèque romande, 1973.

-. L'École contre la vie [I942], dans Pamphlets pédagogiques, Lausanne, L’Âge d'homme, I984, pp. 75-II5.

Jakubec, Doris, " Roorda et les Cahiers vaudois », dans Henri Roorda, Le Pédagogue n'aime pas les enfants, Lausanne, HumuS/Association des amis de Henri Roorda, 20I7, pp. II9-I32.

L'Aminot, Tanguy (éd.), Dossier « Émile et l'éducation », Études Jean-Jacques Rousseau, nº 9, 1997, pp. 217-245.

—. «Henri Roorda, pédagogue rousseauiste et libertaire », dans Marianne Enckell et al., Henri Roorda et l'humour zèbre, Lausanne, HumuS/Association des amis de Henri Roorda, 2009, pp. 6I-78.

Lenoir, Hugues, Henri Roorda ou le zèbre pédagogue, Paris, Le Monde libertaire, 2009.

Losseroy, Gilles, « Roorda, anarchistes de père en fils », dans Marianne Enckell et al., Henri Roorda et l'humour zèbre, Lausanne, HumuS/Association des amis de Henri Roorda, 2009, pp. 39-59.

Mole, Frédéric, L'École laïque pour une République sociale. Controverses pédagogiques et politiques (I90o-I9I4), Rennes, PUR, 2010.

-. "Contextes d'un procès de l'instruction publique », "Union, Étude »: Denis de Rougemont, dir. Nicolas Stenger, François Saint-Ouen et Jonathan Wenger, Revue historique neuchâteloise, $\mathrm{n}^{\circ} \mathrm{I}-2,2019$, pp. 63-69.

Prost, Antoine, Histoire des réformes de l'éducation. De 1936 à nos jours, Paris, Seuil, 2013.

Reclus, Élisée, L’Avenir de nos enfants, Lille, Lagache, I886.

Roorda, Henri, Euvres complètes, éd. André Guex, Lausanne, L'Âge d'homme, 1969 (t. I) et 1970 (t. II).

-. «L'École et l'apprentissage de la docilité », L'Humanité nouvelle, n ${ }^{\circ} 2-\mathrm{I} 3$, I898, repris dans Le Supplément littéraire des Temps nouveaux, $\mathrm{n}^{\circ}$ 36-49, I898, réédité dans Études Jean-Jacques Rousseau, nº 9, I997, pp. 219-245.

- Le Pédagogue n'aime pas les enfants, Lausanne, HumuS/Association des amis de Henri Roorda, 2017 [I9I7]. 
Rougemont, Denis de, Les Méfaits de l'instruction publique [1929] et Suite des méfaits [1972], dans Pamphlets pédagogiques, Lausanne, L'Âge d'homme, I984, pp. II7-I6o et pp. I6I-I75.

Tapernoux, Marc, "Hommage à la mémoire de Henri Roorda ", La Revue, 28 et 29 décembre 1945, réédité dans Henri Roorda, Le Pédagogue n’aime pas les enfants, Lausanne, HumuS/Association des amis de Henri Roorda, 20I7, pp. I35-I4I. 